\title{
ON A SEPARATION THEOREM FOR THE ZEROS OF THE ULTRASPHERICAL POLYNOMIALS
}

\author{
L. H. KANTER
}

1. It will be recalled that the ultraspherical polynomials are those which are orthogonal on the interval $(-1,1)$, corresponding to the weight function $\left(1-x^{2}\right)^{\lambda-1 / 2}, \lambda>-1 / 2$. In what follows $\lambda=0$ will also be excluded. The coefficients of these polynomials are functions of the parameter $\lambda$ appearing in the weight function, and the symbol $P_{n}(x, \lambda)$, indicative of this fact, will be used to denote the ultraspherical polynomial of degree $n$.

This paper is mainly concerned with a separation theorem for the zeros of the ultraspherical polynomials and the zeros of the polynomials derived from the ultraspherical polynomials by differentiation with respect to the parameter $\lambda$, under various conditions of normalization. In addition some generalizations to other related polynomials are obtained, and an application is made also to a limited class of the Jacobi polynomials, which include the ultraspherical polynomials as special cases.

To facilitate the exposition certain relations are listed below for reference. These are taken from Orthogonal polynomials, Gabor Szegö, Amer. Math. Soc. Colloquium Publications, vol. 23, New York, 1939. The first number in the reference bracket will denote the page, and the second number the relation, in the work just mentioned.

$$
P_{n}(x, \lambda)=(-1)^{n} P_{n}(-x, \lambda)
$$

$$
-\ln \left(1-2 x w+w^{2}\right)=\sum_{n=1}^{\infty}(2 / n) T_{n}(x) w^{n}
$$

$$
x_{\nu}^{\prime}(\lambda) \gtrless 0 \text { according as } x_{\nu}(\lambda) \lessgtr 0
$$

$$
\sum_{n=0}^{\infty} P_{n}(x, \lambda) w^{n}=\left(1-2 x w+w^{2}\right)^{-\lambda}
$$

2. Let $R_{n}(x, \lambda)$ be defined by

$$
R_{n}(x, \lambda)=\frac{\partial}{\partial \lambda} P_{n}(x, \lambda), \quad \lambda>-1 / 2, \lambda \neq 0 .
$$

Then using (1.1) we see that

Presented to the Society, November 25, 1950, under the title On the zeros of the parametric derivatives of the ultraspherical polynomials; received by the editors February 5, 1953 and, in revised form, March 6, 1953. 


$$
R_{n}(x, \lambda)=(-1)^{n} R_{n}(-x, \lambda) .
$$

Consequently, the real zeros of $R_{n}(x, \lambda)$ are symmetrically situated in pairs with respect to the origin along the $x$-axis. (For $n$ odd, $x=0$ is a zero.)

Next if we denote the $\nu$ th zero of $P_{n}(x, \lambda)$ by $x_{\nu}(\lambda)$, we have, since $P_{n}\left(x_{v}, \lambda\right)$ is identically zero in $\lambda$,

$$
\left(\frac{\partial P_{n}}{\partial \lambda}\right)_{x=x_{v}}+\left(\frac{\partial P_{n}}{\partial x}\right)_{x=x_{y}} x_{p}^{\prime}(\lambda)=0
$$

or

$$
R_{n}\left(x_{v}, \lambda\right)+P_{n}^{\prime}\left(x_{v}, \lambda\right) x_{v}^{\prime}(\lambda)=0 .
$$

From the reality and simplicity of the zeros of $P_{n}(x, \lambda)$, and from (1.3) above, it is seen that $R_{n}(x, \lambda)$ alternates in sign at successive positive (negative) zeros of $P_{n}(x, \lambda)$. Hence at least $n-2$ zeros of $R_{n}(x, \lambda)$ are real and, by symmetry, half this number is located between successive positive zeros of $P_{n}(x, \lambda)$, one zero to an interval, and a similar statement holds for the negative zeros.

Let us now consider the nature of the remaining two zeros. (We assume that the coefficient $k_{n}$ of $x^{n}$ in $P_{n}(x, \lambda)$ is not a constant, i.e. the polynomial $P_{n}(x, \lambda)$ has been normalized so that its leading coefficient contains $\lambda$.) Because of the reality of the coefficients of $R_{n}(x, \lambda)$, these two zeros are either conjugate imaginary, or else by virtue of (2.2), if both are real, they are either both 0 or else equal in magnitude and opposite in sign.

Moreover, these two zeros cannot be found in any interval between successive zeros where the other $n-2$ zeros individually lie. Because of symmetry it is sufficient to discuss the case of the positive zero. Since $R_{n}(x, \lambda)$ takes opposite signs at consecutive positive zeros of $P_{n}(x, \lambda)$, it must have an odd number of zeros in such an interval because of the real nature of its coefficients. But since one positive zero is unaccounted for, at most, $R_{n}(x, \lambda)$ has only one zero in such an interval, and the statement is seen to be true.

Here let us supplement our results with an expression of $R_{n}(x, \lambda)$ in terms of $P_{n}(x, \lambda)$ and $T_{n}(x)$, where $T_{n}(x)$ is the particular ultraspherical polynomial known as the Tchebychev polynomial of the first kind, that is, the ultraspherical polynomial corresponding to the weight function $\left(1-x^{2}\right)^{-1 / 2}$.

Differentiating (1.4) with respect to $\lambda$, there results

$$
\sum_{n=1}^{\infty} R_{n}(x, \lambda) w^{n}=-\left(1-2 x w+w^{2}\right)^{-\lambda} \ln \left(1-2 x w+w^{2}\right) .
$$


Then with the aid of (1.2) we get

$$
\sum_{n=1}^{\infty} R_{n}(x, \lambda) w^{n}=\sum_{n=1}^{\infty}\left(\frac{2}{n}\right) T_{n}(x) w^{n} \sum_{n=0}^{\infty} P_{n}(x, \lambda) w^{n} .
$$

The Cauchy product then gives us

$$
R_{n}(x, \lambda)=\frac{\partial}{\partial \lambda} P_{n}(x, \lambda)=2 \sum_{\nu=0}^{n-1} \frac{P_{\nu}(x, \lambda) T_{n-\nu}(x)}{n-\nu} .
$$

The difficulties encountered with the two zeros of $R_{n}(x, \lambda)$ do not occur in case we consider polynomials defined by $S_{n-2}$ $=\partial\left\{k_{n}^{-1} P_{n}\right\} / \partial \lambda$, because these polynomials are of degree $n-2$, as our notation has already anticipated. $\left(k_{n}(\lambda)\right.$ is the coefficient of $x^{n}$ in $P_{n}(x, \lambda)$.)

Since $\partial\left\{k_{n}^{-1} P_{n}\right\} / \partial \lambda=P_{n} \partial\left(k_{n}^{-1}\right) / \partial \lambda+k_{n}^{-1} R_{n}(x, \lambda)$, we need but use the information obtained in connection with $R_{n}(x, \lambda)$ to summarize our results in

TheOREM 2.1. The positive (negative) zeros of $R_{n}(x, \lambda)$ interlace, or alternate, with the positive (negative) zeros of $P_{n}(x, \lambda) . A l s o$,

$$
R_{n}(x, \lambda)=2 \sum_{\nu=0}^{n-1} P_{\nu}(x, \lambda) \frac{T_{n-\nu}(x)}{n-\nu} .
$$

The positive (negative) zeros of $S_{n-2}(x, \lambda)$ interlace, or alternate, with the positive (negative) zeros of $P_{n}(x, \lambda)$, and all the zeros of $S_{n-2}(x, \lambda)$ are real.

3. In this section we treat of some polynomials constructed from those in the previous section.

Consider the polynomials $V_{n}(x, \lambda) \equiv R_{n}(x, \lambda)+c(\lambda) P_{n}(x, \lambda)$, where $c(\lambda)$ is arbitrary and real. Clearly, the signs of these polynomials at the zeros of $P_{n}(x, \lambda)$ are the same as the signs of $R_{n}(x, \lambda)$ at these zeros. Hence $n-2$ zeros are immediately accounted for as in the previous section.

Next, let $k_{\nu}(\lambda)$ and $k_{\nu}^{\prime}(\lambda)$ denote the corresponding coefficients of $x^{\nu}, \nu=0,1,2, \cdots, n$, in $P_{n}(x, \lambda)$ and $R_{n}(x, \lambda)$, respectively. Also, let $x_{n}$ and $x_{1}$ denote the greatest and least positive zeros of $P_{n}(x, \lambda)$, and suppose, for the sake of definiteness, that $k_{n}>0$.

Then the zeros of $V_{n}(x, \lambda)$ may be described by

THEOREM 3.1. $V_{n}(x, \lambda)$ will have all of its zeros real if either

$$
k_{n}^{\prime}(\lambda) / k_{n}(\lambda)+c(\lambda) \leqq 0,
$$




$$
k_{0}^{\prime}(\lambda) / k_{0}(\lambda)+c(\lambda) \geqq 0, \quad \text { or } \quad k_{1}^{\prime}(\lambda) / k_{1}(\lambda)+c(\lambda) \geqq 0,
$$

\section{according as $n$ is even or odd.}

If case (i) obtains, then $V_{n}(x, \lambda)$ will have a positive zero greater than $x_{n}$ of $P_{n}(x, \lambda)$, or $V_{n}(x, \lambda)$ will be of degree $n-2$, according as the inequality or equality sign holds.

Should case (ii) obtain, then $V_{n}(x, \lambda)$ will have a positive zero in $\left(0, x_{1}\right)$, or a double or triple zero at the origin according as the inequality or equality signs hold, the double zero pertaining to the case $n$ even, the triple zero to the case $n$ odd.

Corresponding statements can of course be made for the negative zero from considerations of symmetry.

If neither of these conditions is satisfied, then $V_{n}(x, \lambda)$ will have a pair of conjugate imaginary zeros.

The reasoning is as follows. Since $k_{n}(\lambda)>0, P_{n}^{\prime}\left(x_{n}, \lambda\right)>0$. Then using (2.3) we see that $R_{n}\left(x_{n}, \lambda\right)$ is positive, and hence also $V_{n}\left(x_{n}, \lambda\right)$ $>0$. So considering $V_{n}(x, \lambda)$ for large values of $x$ in connection with case (i), we see that the statement in the theorem is true for the inequality sign. If equality prevails, obviously $V_{n}(x, \lambda)$ is of degree $n-2$.

Now consider case (ii) with $n$ even and the inequality sign holding. Suppose first that $k_{0}(\lambda)>0$. Then $P_{n}^{\prime}\left(x_{1}, \lambda\right)<0$, and (2.3) shows this time that $R_{n}\left(x_{1}, \lambda\right)<0$. Hence so is $V_{n}\left(x_{1}, \lambda\right)$. From our assumption it follows that $V_{n}(0, \lambda)$ is positive, and the theorem is thus verified for this case. The statement for the equality sign is again easily verified, and it is readily seen that if $k_{0}(\lambda)<0$, the statement still holds.

The second part of case (ii), that is, when $n$ is odd, may be treated as follows. Evidently, the triple root at the origin occurs for $k_{1}^{\prime} / k_{1}+c$ $=0$, so we consider the case $k_{1}^{\prime} / k_{1}+c>0$.

If $n$ is odd, then $P_{n}(x, \lambda)$ is of the form $x Q_{n-1}(x, \lambda)$ and $R_{n}(x, \lambda)$ is of the form $x S_{n-1}(x, \lambda)$, with $V_{n}(x, \lambda)$ of the form $x T_{n-1}(x, \lambda)$, where $T_{n-1}(x, \lambda) \equiv S_{n-1}(x, \lambda)+c Q_{n-1}(x, \lambda)$. Further, $P_{n}^{\prime}\left(x_{\nu}, \lambda\right)=x_{\nu} Q_{n-1}^{\prime}\left(x_{\nu}, \lambda\right)$.

Suppose first that $k_{1}>0$. Then $Q_{n-1}^{\prime}\left(x_{1}, \lambda\right)<0$, and so is $P_{n}^{\prime}\left(x_{1}, \lambda\right)$. From (2.3) we find that $R_{n}\left(x_{1}, \lambda\right)<0$, and hence so is $T_{n-1}\left(x_{1}, \lambda\right)$. It then follows that $T_{n-1}(x, \lambda)$, and hence $V_{n}(x, \lambda)$ has a root in $\left(0, x_{1}\right)$. If $k_{1}<0$, the same conclusion follows.

The statement about the imaginary zeros follows from the fact that at most one positive root is in question coupled with the fact that we are concerned with polynomials having real coefficients.

4. In this last section we make an application to the Jacobi polynomials, which, it will be recalled, are orthogonal to each other on $(-1,1)$, and which correspond to the weight function 
$(1-x)^{\alpha}(1+x)^{\beta}, \alpha, \beta>-1$. The zeros of these polynomials are all real, simple, and lie in the interior of $(-1,1)$.

Let the polynomials $R_{n-1}(x, \lambda)$ be defined by

$$
R_{n-1}(x, \lambda)=\frac{\partial}{\partial \lambda}\left[k_{n}^{-1} P_{n}(x, \alpha, \beta)\right]=\frac{\partial}{\partial \lambda}\left[k_{n}^{-1} P_{n}(x, \lambda)\right],
$$

where $\alpha=\alpha(\lambda)>-1, \beta=\beta(\lambda)>-1$.

Proceeding as in $\$ 2$, we obtain

$$
R_{n-1}\left(x_{\nu}, \lambda\right)+k_{n}^{-1} P_{n}^{\prime}\left(x_{v}, \lambda\right)\left[\frac{\partial x_{v}}{\partial \alpha} \alpha^{\prime}(\lambda)+\frac{\partial x_{\nu}}{\partial \beta} \beta^{\prime}(\lambda)\right]=0 .
$$

If we now demand that $\alpha^{\prime}(\lambda) \cdot \beta^{\prime}(\lambda) \leqq 0,\left|\alpha^{\prime}(\lambda)\right|+\left|\beta^{\prime}(\lambda)\right|>0$, and then use the fact that $\partial x_{v} / \partial \alpha<0, \partial x_{\nu} / \partial \beta>0$ (see $[117 ;(6.21 .2)]$ ), we have

TheOREM 4.1. All the zeros of $R_{n-1}(x, \lambda)$, with the parameters $\alpha, \beta$ so restricted that $\alpha=\alpha(\lambda)>-1, \beta=\beta(\lambda)>-1, \alpha^{\prime}(\lambda) \cdot \beta^{\prime}(\lambda) \leqq 0,\left|\alpha^{\prime}(\lambda)\right|$ $+\left|\beta^{\prime}(\lambda)\right|>0$, interlace with the zeros of $P_{n}(x, \alpha, \beta)=P_{n}(x, \lambda)$, and all lie in the interior of $(-1,1)$.

To illustrate the theorem we mention the polynomials $R_{n-1}(x, \lambda)$ derived from the Jacobi polynomials by taking $\alpha=\lambda, \beta=0$, and then $\beta=\lambda, \alpha=0$ in the weight function-that is, the polynomials deriving from the Jacobi polynomials with weight functions $(1-x)^{\lambda}$ and $(1+x)^{\lambda}$, respectively, with $\lambda>-1$.

Mississippi State College 